\title{
New test of Lorentz symmetry using ultrahigh-energy cosmic rays
}

\author{
Luis A. Anchordoqui ${ }^{1,2,3}$ and Jorge F. Soriano ${ }^{1,2}$ \\ ${ }^{1}$ Department of Physics and Astronomy, Lehman College, City University of New York, \\ New York 10468, USA \\ ${ }^{2}$ Department of Physics, Graduate Center, City University of New York, New York 10016, USA \\ ${ }^{3}$ Department of Astrophysics, American Museum of Natural History, New York 10024, USA
}

(Received 9 November 2017; published 12 February 2018)

\begin{abstract}
We propose an innovative test of Lorentz symmetry by observing pairs of simultaneous parallel extensive air showers produced by the fragments of ultrahigh-energy cosmic ray nuclei which disintegrated in collisions with solar photons. We show that the search for a cross-correlation of showers in arrival time and direction becomes background free for an angular scale $\lesssim 3^{\circ}$ and a time window $\mathcal{O}(10 \mathrm{~s})$. We also show that if the solar photo-disintegration probability of helium is $\mathcal{O}\left(10^{-5.5}\right)$ then the hunt for spatiotemporal coincident showers could be within range of existing cosmic ray facilities, such as the Pierre Auger Observatory. We demonstrate that the actual observation of a few events can be used to constrain Lorentz violating dispersion relations of the nucleon.
\end{abstract}

DOI: $10.1103 /$ PhysRevD.97.043010

Ever since Greisen, Zatsepin, and Kuzmin (GZK) pointed out that the pervasive radiation fields make the Universe opaque to the propagation of ultrahigh-energy $\left(E \gtrsim 10^{9} \mathrm{GeV}\right.$ ) cosmic rays (UHECRs) $[1,2]$, it became evident that the actual observation of the GZK effect would provide strong constraints on Lorentz invariant breaking effects. This is because if Lorentz invariance is broken in the form of nonstandard dispersion relations for various particles, then absorption and energy loss processes for UHECR interactions would be modified; see e.g. [3-11]. In particular, the GZK interactions (photo-pion production and nucleus photo-disintegration) are characterized by well defined energy thresholds [near the excitation of the $\Delta^{+}(1232)$ and the giant dipole resonance, respectively], which can be predicted on the basis of Lorentz invariance. Therefore, the experimental confirmation that UHECR processes occur at the expected energy thresholds can be considered as an indirect piece of evidence supporting Lorentz symmetry under colossal boost transformations.

A suppression in the UHECR flux at $E \gtrsim 10^{10.6} \mathrm{GeV}$ has been established beyond no doubt by the HiRes [12], Auger [13], and Telescope Array (TA) [14] experiments. By now (in Auger data) the suppression has reached a statistical significance of more than $20 \sigma$ [15]. This suppression is consistent with the GZK prediction that interactions with

Published by the American Physical Society under the terms of the Creative Commons Attribution 4.0 International license. Further distribution of this work must maintain attribution to the author(s) and the published article's title, journal citation, and DOI. Funded by SCOAP . universal photon fields will rapidly degrade the energy of UHECRs. Intriguingly, however, there are also indications that the source of the suppression may be more complex than originally anticipated.

Observations of the rate of change with energy of the mean depth-of-shower-maximum $X_{\max }$ seem to indicate that the cosmic ray composition becomes lighter as energy increases toward $E \sim 10^{9.3} \mathrm{GeV}$ from below [16], fueling a widespread supposition that extragalactic cosmic rays are primarily protons. However, Auger high-quality, highstatistics data, when interpreted with the leading LHCtuned shower models, exhibit a strong likelihood for a composition that becomes gradually heavier with increasing energy; namely, $1.5 \lesssim\langle\ln A\rangle \lesssim 3$, for $10^{9.5} \lesssim E \lesssim 10^{10.6}$ [17-20]. Within uncertainties, the data from TA are consistent with these findings [21,22]. For $E \gtrsim 10^{10.6} \mathrm{GeV}$, the indication of an anisotropy at an intermediate angular scale of $13^{\circ}$ (significant at the $4.0 \sigma$ level [23]) [24] points to a similar nuclear composition. Note that for $E / Z=10^{10} \mathrm{GeV}$, typical deflections of UHECRs crossing the Galaxy are about $10^{\circ}$, where $Z e$ is the nucleus charge [25].

For a uniform source distribution, the simultaneous fit to the UHECR spectrum and composition $\left(X_{\max }\right.$ and its fluctuations) imposes severe constraints on model parameters: (i) hard source spectra and (ii) a maximum acceleration energy $E_{\max } \lesssim 10^{9.7} Z \mathrm{GeV}$ [26-28]. Hence, under the assumption of a uniform source distribution, the data seem to favor the so-called "disappointing model" [29] wherein it is postulated that the "end-of steam" for cosmic accelerators is coincidentally near the putative GZK cutoff, with the exact energy cutoff determined by data. This interpretation encompasses a radically different viewpoint 
in which the maximum energy of the most powerful cosmic ray accelerators would be observed for the first time and therefore could call into question limits on the violation of Lorentz invariance deduced using the observed suppression in the UHECR spectrum [30-32].

Very recently, one of us put forward a multidimensional reconstruction of the individual emission spectra (in energy, arrival direction, and nuclear composition) to study the hypothesis that primaries are heavy nuclei subject to GZK photo-disintegration and to determine the nature of the extragalactic sources [33]. In this paper we introduce an alternative approach to probe Lorentz invariance using UHECRs. We propose to search for a cross-correlation in arrival time and direction of the secondary nucleon (of energy $E / A$ ) produced via photo-disintegration of an UHECR nucleus (of energy $E$ and baryon number $A$ ) and the associated surviving fragment (of baryon number $A-1$ ). Such a correlation study is possible because: (i) the Lorentz factor (which is equivalent to energy per nucleon) is conserved for photo-disintegration and (ii) the trajectory of cosmic rays within a magnetic field is only rigiditydependent; the relevant quantity for the separation among fragments (hereafter identified with subindices 1 and 2) is $\left|Z_{1} / A_{1}-Z_{2} / A_{2}\right|$.

A simple dimensional argument constrains the distance to the photo-disintegration site. Assuming the energy difference between nucleons inside the nucleus is given by the binding energy $E_{0} \sim \mathrm{MeV}$, the difference in velocity of the secondary products is

$$
\delta v=\sqrt{2 E_{0} / M} \sim \sqrt{10^{-3} / A},
$$

where $M \simeq A \mathrm{GeV}$ is the mass of the parent nucleus. The difference in the time of flight of the secondary products is then

$$
\delta t \sim \delta L=\frac{(L / \mathrm{Mpc})}{\gamma} \delta v \times 10^{24} \mathrm{~cm},
$$

where $L$ is the distance to the photo-disintegration site and $\gamma(=E / M$ at Earth $)$ contracts this length. For a simultaneous observation of the two secondaries at Earth, we demand $\delta L \lesssim 2 R_{\oplus}\left(\sim 10^{9} \mathrm{~cm}\right)$, which yields

$$
\gamma \sim \frac{10^{14}}{\sqrt{10 A}}(L / \mathrm{Mpc}) .
$$

For the particular range $10^{9} \lesssim \gamma \lesssim 10^{10}$, which spans the UHECR spectrum, (3) constrains the photo-disintegration site to a distance $\lesssim \mathrm{kpc}$. It has long been known that UHECR nuclei scattering off the universal radiation fields have a mean free path $\gg$ kpc [34]. Moreover, we know the devil is in the detail, and so the number of GZK interactions which would lead to a simultaneous observation of their secondary products on Earth is essentially negligible.
Of particular interest here, UHECR nuclei en route to Earth also interact with the solar radiation field and photodisintegrate $[35,36]$. The nuclear photo-disintegration process has two characteristic regimes. There is the domain of the giant dipole resonance (GDR), where a collective nuclear mode is excited with the subsequent emission of one (or possibly two nucleons), and the high energy plateau, where the excited nucleus decays dominantly by two nucleon and multinucleon emission. The energy range of the GDR in the nucleus rest frame spans $10 \lesssim \varepsilon^{\prime} / \mathrm{MeV} \lesssim 30$, and the plateau extends up to the photo-pion production threshold (i.e., photon energy $\varepsilon^{\prime} \sim 150 \mathrm{MeV}$ ).

The background radiation field can be described by a Planckian spectrum, with a temperature of the solar surface $T_{s} \simeq 0.5 \mathrm{eV}$, normalized to reproduce the solar luminosity, $L_{\odot}=4 \pi r^{2} c \int d \varepsilon \varepsilon d n / d \varepsilon$, yielding

$\frac{d n}{d \varepsilon}=7.2 \times 10^{7} \frac{\varepsilon^{2}}{\exp \left(\varepsilon / T_{s}\right)-1}\left(\frac{r}{\mathrm{AU}}\right)^{-2}(\mathrm{eV} \mathrm{cm})^{-3}$,

where $r$ is the spherical radial coordinate centered at the Sun. In the rest frame of the nucleus, the energy $\varepsilon$ of the solar photons (in the rest frame of the Sun) is highly blueshifted to

$$
\varepsilon^{\prime}=\varepsilon \gamma(1+\beta \cos \alpha) \sim 2 \gamma \varepsilon c_{\alpha / 2}^{2},
$$

where $\beta=\sqrt{1-1 / \gamma^{2}} \sim 1$ and $c_{\alpha / 2}=\cos (\alpha(\ell) / 2)$, and where $\alpha(\ell)$ is the angle between the momenta of photon and nucleus in the Sun's reference frame, with $\ell$ the coordinate along the path of the nucleus; i.e., $\cos \alpha=\hat{\ell} \cdot \hat{r}$.

The GDR cross section in the narrow width approximation is

$$
\sigma(\varepsilon)=\frac{\pi}{2} \sigma_{0} \Gamma \delta\left(2 \gamma \varepsilon c_{\alpha / 2}^{2}-\varepsilon_{0}\right),
$$

where $\Gamma$ and $\sigma_{0}$ are the GDR width and cross section at maximum; the factor of $1 / 2$ is introduced to match the integral (i.e., total cross section) of the Breit-Wigner and the delta function [37]. Fitted numerical formulas are $\sigma_{0}=1.45 A \mathrm{mb}, \Gamma=8 \mathrm{MeV}$, and $\varepsilon_{0}=42.65 A^{-0.21} \mathrm{MeV}$ for $A>4$ and $\varepsilon_{0}=0.925 A^{2.433} \mathrm{MeV}$ for $A \leq 4$ [38]. In the high energy regime the cross section is well approximated by $\sigma(\varepsilon) \approx A / 8 \mathrm{mb}$.

All in all, the probability that a nucleus photo-disintegrates on the solar radiation along its path towards the Earth is found to be

$$
\eta_{A}=1-\exp \left[-\int_{0}^{\infty} d \ell \frac{1}{\lambda(\ell)}\right]
$$

where 


$$
\frac{1}{\lambda(\ell)}=\int_{0}^{\infty} \sigma(\varepsilon) \frac{d n}{d \varepsilon} 2 c_{\alpha / 2}^{2} d \varepsilon
$$

is the inverse photo-disintegration mean-free-path [39]. Integration of (7) yields $10^{-5} \lesssim \eta_{A} \lesssim 10^{-4}$ for iron, and $10^{-6} \lesssim \eta_{A} \lesssim 10^{-5}$ for helium and oxygen. These values of $\eta_{A}$ are in agreement with the estimates in [39-41].

Since the secondary fragments have slightly different rigidities the deflection in the interplanetary magnetic field results in two separate extensive air showers, arriving essentially at the same time and from the same direction in the sky $[39,40]$. More specifically, the average separation of the shower on Earth can be parametrized by [41]

$$
\langle\delta L\rangle_{A}=4 A\left|\frac{Z_{1}}{A_{1}}-\frac{Z_{2}}{A_{2}}\right|\left(\frac{E}{10^{10} \mathrm{GeV}}\right)^{-1} \mathrm{~km},
$$

where $E$ is the energy of the parent nucleus. The average separation of showers as estimated in [40] is somewhat smaller. For a given experiment, each nuclear species has a critical energy above which $\langle\delta L\rangle_{A}$ would be comparable to the size of the instrumented area. As a benchmark we consider a $3000 \mathrm{~km}^{2}$ array of detectors, with interspacing of about $1.5 \mathrm{~km}$. For ${ }^{4} \mathrm{He}$, (9) yields $\langle\delta L\rangle_{\mathrm{He}} \sim 50 \mathrm{~km}$ at $E \sim 10^{9.3} \mathrm{GeV}$. However, for ${ }^{56} \mathrm{Fe}$, at the same energy (9) leads to $\langle\delta L\rangle_{\mathrm{Fe}} \sim 260 \mathrm{~km}$, and so the separation distance between the showers would be out of detection range.

Because the intensity of cosmic rays is steeply falling with energy, contributions from the counting rate at the critical energy dominate the integrated event rate. Existing estimates of the event rate at UHECR facilities [39-42] are subject to large uncertainties, mainly because $\eta_{A}$ and $\langle\delta L\rangle_{A}$ depend strongly on $A$ and the nuclear composition of UHECRs is poorly known.

Herein, we assume a nuclear composition dominated by helium at $E \gtrsim 10^{9.3} \mathrm{GeV}$ that becomes gradually heavier with increasing energy; see e.g. Fig. 4 of [27]. We further assume that the photo-disintegration probability of helium on the solar photons is $\eta_{\mathrm{He}} \sim 10^{-5.5}$. These two assumptions together lead to an expected integrated flux of

$\frac{d F}{d t d \Omega d A}\left(E>10^{9.3} \mathrm{GeV}\right) \sim 3 \times 10^{-5} \mathrm{~km}^{-2} \mathrm{sr}^{-1} \mathrm{yr}^{-1}$,

where $E$ denotes the energy of the parent nucleus. This flux is in agreement with the one shown in Fig. 3 of [40]. Moreover, as exhibited in Fig. 2 of [40], for $\eta_{\mathrm{He}} \sim 10^{-5.5}$ and $E \gtrsim 10^{9} \mathrm{GeV}$, we have $20 \lesssim\langle\delta L\rangle_{\mathrm{He}} / \mathrm{km} \lesssim 50$. The flux derived herein, using a helium saturated spectrum above $10^{9.3} \mathrm{GeV}$, is larger than the intensity derived in [41] using the spectrum of [43]. Whichever flux calculation one may find more convincing, it seems most conservative at this point to depend on experiment (if possible) to resolve the issue.
The $3000 \mathrm{~km}^{2}$ surface detector array of the Pierre Auger Observatory is fully efficient at $E \gtrsim 10^{9.5} \mathrm{GeV}$ [44]. From January 2004 until December 2016 this facility has accumulated an exposure [24]

$$
\mathcal{E}\left(E>10^{9.5} \mathrm{GeV}\right)=6.7 \times 10^{4} \mathrm{~km}^{2} \mathrm{sr} \mathrm{yr} .
$$

At lower energies, the trigger efficiency of the surface detector array decreases smoothly and becomes roughly $30 \%$ at $10^{8.7} \mathrm{GeV}$ [44]. To get a rough estimate of the exposure available to probe spatiotemporal correlations of air showers in an experiment like Auger we scale down $\mathcal{E}\left(E>10^{9.5} \mathrm{GeV}\right)$ by a factor of 0.3 . This leads to

$$
\mathcal{E}\left(10^{8.7}<E / \mathrm{GeV}<10^{9.3}\right) \gtrsim 2 \times 10^{4} \mathrm{~km}^{2} \text { sr yr. }
$$

For ${ }^{4} \mathrm{He}, \Delta E=E_{2}-E_{1} \sim 3 \gamma \mathrm{GeV}$. For $E>10^{9.3} \mathrm{GeV}$, (10) and (12) lead to an expected integrated rate which is consistent with one event.

It is clear that for a signal $\mathcal{O}(1)$ event we must learn how to properly conduct background rejection to ascertain whether the observation of a few events is due to physics or statistics. Moreover, to calculate a meaningful statistical significance in the shower cross-correlation analysis, it is important to define the search procedure a priori in order to ensure it is not inadvertently devised especially to suit the particular data set after having studied it. With the aim of avoiding accidental bias on the number of trials performed in selecting the cuts, we now conduct a phenomenological analysis of the potential background to define the angular and temporal cuts.

We start by selecting a reference direction on the sky $\mathfrak{d}_{0}$. We define $\theta$ as the angle between $\mathfrak{D}_{0}$ and the other direction on the sky $\mathfrak{d}$. We define $\phi$ as the angular distance between a reference axis, placed on the normal plane to the vector pointing in the direction $\mathfrak{d}_{0}$, and the projection on that plane of a vector pointing towards $\mathfrak{d}$. With this construction, $\theta \in$ $[0, \pi]$ and $\phi \in[0,2 \pi]$.

The expected fraction of events that will be contained in a cap of the sphere within an angle $\alpha$ to the direction $\mathfrak{b}_{0}$, for all $\phi$, and in a time interval $t$ is

$$
\begin{aligned}
f(\alpha, t) & =\frac{t}{T} \int_{0}^{2 \pi} d \phi \int_{0}^{\alpha} d \theta \frac{1}{4 \pi} \sin \theta \\
& =\frac{1}{2} \frac{t}{T}(1-\cos \alpha),
\end{aligned}
$$

where $T$ is the time span for the experiment. In a sample of $N$ events, we expect $\mu(\alpha, t)=N f(\alpha, t)$ events in the angletime window defined above. The actual number of events in that window are distributed following a Poisson distribution of mean $\mu(\alpha, t)$. The probability of observing $k$ events in an angle-time window is then 

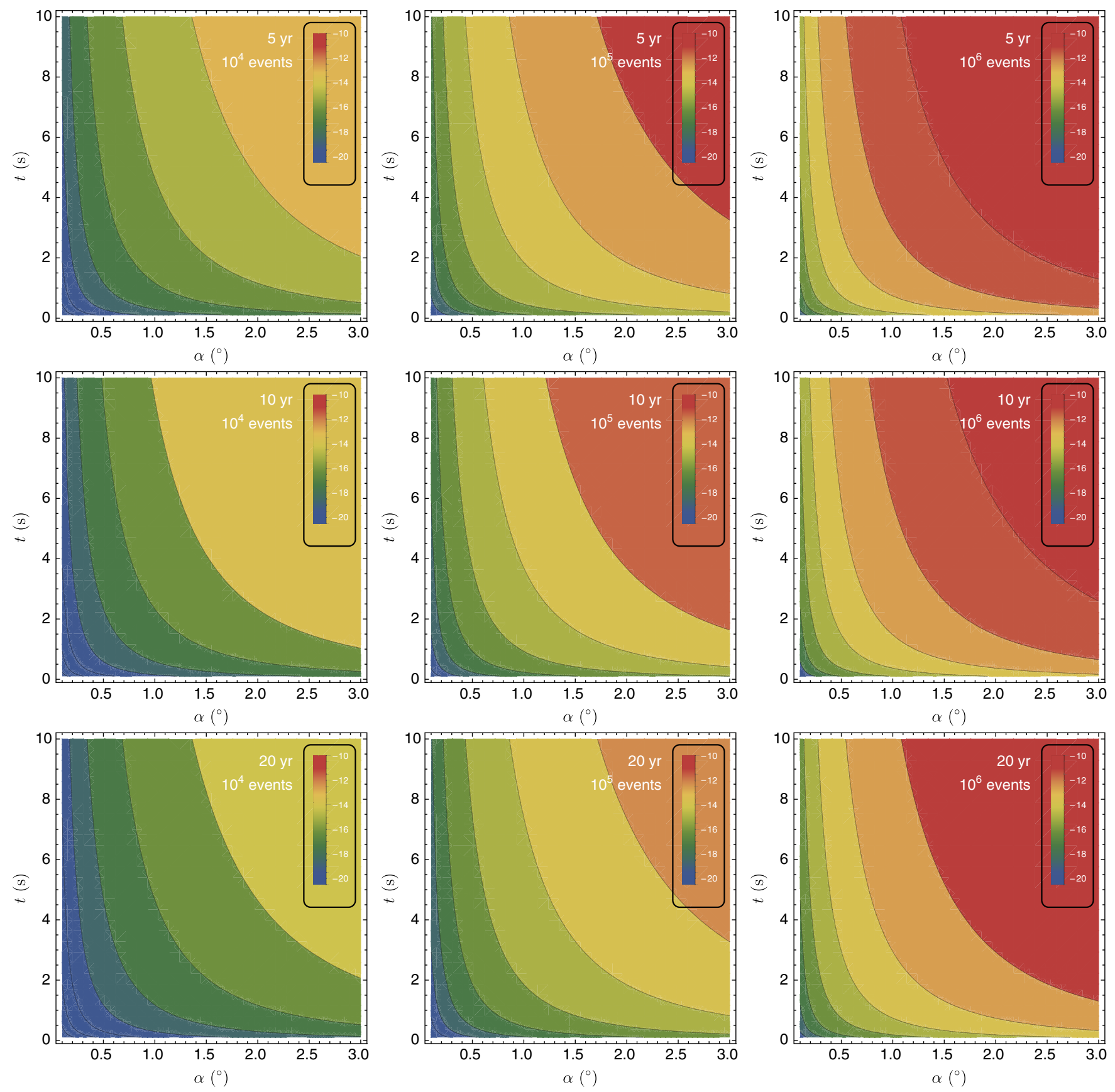

FIG. 1. $\log _{10} p_{2}(\alpha, t)$ for different total number of events (from left to right $N=10^{4}, 10^{5}, 10^{6}$ ), and different lifetimes of the experiment (from top to bottom $T / \mathrm{yr}=5,10,20$ ). $T=10 \mathrm{yr}$ and $T=20 \mathrm{yr}$ are scales compatible with Auger, while $T=5 \mathrm{yr}$ is an approximation for the life span of the prospective experiment POEMMA [45].

$$
p_{k}(\alpha, t)=\frac{e^{-\mu(\alpha, t)} \mu(\alpha, t)^{k}}{k !}
$$

In Fig. 1 we show the function $\log _{10} p_{2}(\alpha, t)$, for $\alpha \in$ $\left[0^{\circ}, 3^{\circ}\right]$ and $t \in[0 \mathrm{~s}, 10 \mathrm{~s}]$, which gives the probability of measuring two events in an angle-time window specified by the pair $(\alpha, t)$. Since

$$
\frac{\sum_{k=3}^{\infty} p_{k}(\alpha, t)}{p_{2}(\alpha, t)} \lesssim 10^{-6}
$$

in our $(\alpha, t)$ range of interest, $p_{2}(\alpha, t)$ practically accounts for the probability of having not only two, but any amount of events above one.

The quantity $p_{2}(\alpha, t)$ is then the $p$-value for observing a coincidence of two detections in a background only 


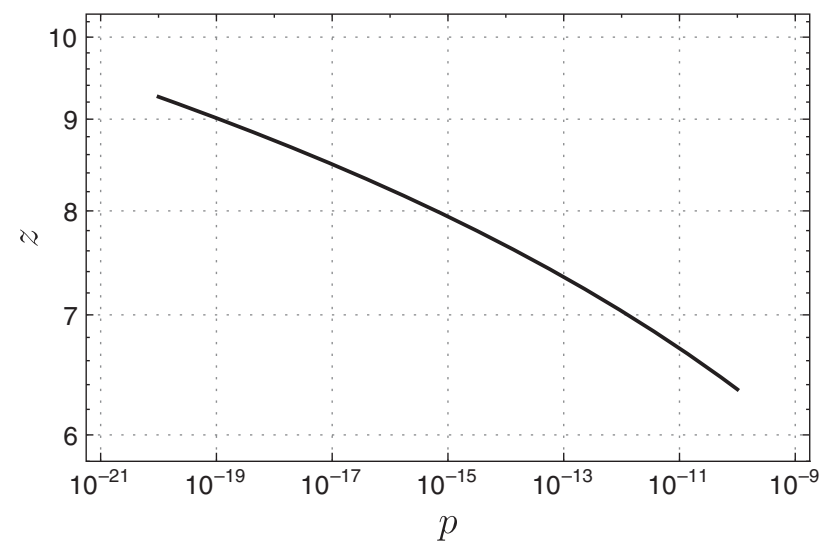

FIG. 2. Relation between the $p$-value and $z$, the number of standard deviations away from the mean, for a normal distribution.

hypothesis. To quantify this in a more comprehensible way, one can use the usual relation between $p$-values and $\sigma$ levels following a normal distribution

$$
p=\frac{1}{2}\left[1-\operatorname{erf}\left(\frac{z}{\sqrt{2}}\right)\right]
$$

being $z$ the number of standard deviations from the mean. The relation between $p$ and $z$ is shown in Fig. 2. One can check by inspection that for the whole range of $\alpha$ and $t$, the $p$-value for observing two or more events together in a small angle-time window is a more than 5-sigma effect against the background. Hence, the actual observation of a few pairs of cross-correlated events would become the smoking gun to set model independent constraints on Lorentz invariance violation.

Strictly speaking, a nucleus with baryon number $A$ and charge $\mathrm{Z} e$ would have a nonstandard dispersion relation of the form

$$
E_{A, Z}^{2}=p_{A, Z}^{2}+M_{A, Z}^{2}+\zeta_{A, Z} \frac{p_{A, Z}^{n+2}}{M_{\mathrm{Pl}}^{n}},
$$

where $E_{A, Z}$ is the nucleus energy, $p_{A, Z}$ is the absolute value of its 3-momentum, and $M_{A, Z}$ its mass. Here, $M_{\mathrm{Pl}} \approx$ $10^{19} \mathrm{GeV}$ is the Planck mass and $\zeta_{A, Z}$ are Lorentz-violating parameters of the nucleus. In the rest frame of the Sun we assume that only baryons have nonstandard dispersion relations (note that the solar photon fields have too low energy for Lorentz invariant breaking effects to be relevant in their dispersion relations), and so one can easily obtain a threshold relation, which constrains the $\zeta_{A, Z}$ coefficients when confronted with data. Actually, since we expect nuclear physics to have negligible Lorentz breaking effects we can write $\zeta_{A, Z}=\zeta / A^{2}$, where $\zeta$ regulates deviations from Lorentz symmetry of the nucleon. The baryon number $A$ of the original disintegrated nucleus can simply be determined by estimating the energies of the primaries of the two air showers, $A=1+E_{2} / E_{1}$, where $E_{1}$ is the energy of the less energetic shower. With the $<20 \%$ energy resolution achieved by the Pierre Auger Observatory [46], the estimation of $A$ is obtained with a resolution $\sigma(A) / A<0.2 \sqrt{2}(1-1 / A)$, which is around $20 \%$ for a helium primary, or $\sigma(A=4) \sim 0.85$, allowing its differentiation from other primaries with $A$ around 4. This provides an univocal (model independent) determination of the nuclear composition and thereupon bounds the threshold energy interval to be compatible with experimental results on photo-nuclear interactions [47-56].

For ${ }^{4} \mathrm{He}$, the photo-excitation cross section of the GDR has a threshold $\varepsilon_{\text {th }}^{\prime} \approx 20 \mathrm{MeV}$ [57]. The GDR decays by the statistical emission of a single nucleon, leaving an excited daughter nucleus $(A-1)^{*}$. The probability for emission of two (or more) nucleons is smaller by an order of magnitude. The excited daughter nuclei typically deexcite by emitting one or more photons of energies $1 \lesssim$ $\epsilon^{\prime} / \mathrm{MeV} \lesssim 5$ in the nuclear rest frame [37]. For simplicity, herein we neglect the de-excitation process and consider the photo-disintegration reaction with two incoming particles (nucleus + photon) and two outgoing particles (nucleus + nucleon). Though we are primarily interested in helium photo-disintegration, the ensuing discussion is framed in a general context. The energy-momentum 4-vectors for the four particles in the rest frame of the Sun are $(E, \mathbf{p})$, for the incoming nucleus; $(\varepsilon, \mathbf{k})$, for the photon; $\left(E_{1}, \mathbf{p}_{1}\right)$, for the nucleon; and $\left(E_{2}, \mathbf{p}_{2}\right)$, for the outgoing nucleus. The relation describing the conservation of energy and momentum is given by

$(E+\varepsilon)^{2}-(\mathbf{p}+\mathbf{k})^{2}=\left(E_{1}+E_{2}\right)^{2}-\left(\mathbf{p}_{1}+\mathbf{p}_{2}\right)^{2}$.

We are interested in studying the energy thresholds for which the relation (18) holds.

According to the threshold theorem, "at an upper or lower threshold the incoming particle momenta are always anti-parallel and the final particle momenta are parallel" [58]. This applies for dispersion relations $E(\mathbf{p})$ depending on $p \equiv|\mathbf{p}|$, and being a monotonically increasing function of that variable, when energy and momentum are conserved additive quantities. Then, to obtain the threshold conditions, one can make use of $\mathbf{p} \cdot \mathbf{k}=-p k$ and $\mathbf{p}_{1} \cdot \mathbf{p}_{2}=p_{1} p_{2}$. Since we neglect Lorentz invariant breaking effects on the solar photon fields we take $\varepsilon=k$. In threshold conditions the reaction is collinear, and so $p-k=p_{1}+p_{2}$. Since $k$ is much smaller than the other momenta, we have $p \approx p_{1}+p_{2}$. Following [10], we define $p_{2}=\varkappa p$ and $p_{1}=(1-\varkappa) p$, with $0<\varkappa<1$. Now, neglecting the mass difference between the proton and the neutron $\left(M_{A, Z}=A m_{p}\right.$, where $m_{p}$ is the proton mass), the energy conservation relation is found to be 
$\xi_{A}(1)+\frac{2 \varepsilon}{p}\left[1+\sqrt{1+\xi_{A}(1)}\right]=\varkappa^{2} \xi_{A-1}(\varkappa)+(1-\varkappa)^{2} \xi_{1}(1-\varkappa)+2 \varkappa(1-\varkappa)\left[\sqrt{1+\xi_{A-1}(\varkappa)} \sqrt{1+\xi_{1}(1-\varkappa)}-1\right]$,

where

$$
\xi_{A}(\varkappa)=\left(\frac{A m_{p}}{\varkappa p}\right)^{2}+\frac{\zeta}{A^{2}}\left(\frac{\varkappa p}{M_{\mathrm{Pl}}}\right)^{n}
$$

Note that for $M_{A, Z} \ll p_{A, Z} \equiv p \ll M_{\mathrm{Pl}}, \quad \xi_{A}(\varkappa) \ll 1$. Expanding the square roots to first order in the $\xi$ functions,

$$
\left(1+\frac{\varepsilon}{p}\right) \xi_{A}(1)+\frac{4 \varepsilon}{p}=\varkappa \xi_{A-1}(\varkappa)+(1-\varkappa) \xi_{1}(1-\varkappa) .
$$

Since all the $\xi$-functions are of the same order and $\varepsilon \ll p$, the term $\varepsilon \xi_{A}(1) / p$ is negligible in comparison to the rest of the terms, and so (21) becomes

$$
\xi_{A}(1)+4 \frac{\varepsilon}{p}=\varkappa \xi_{A-1}(\varkappa)+(1-\varkappa) \xi_{1}(1-\varkappa) .
$$

After some algebra, (22) can be rewritten as

$$
\zeta g(\varkappa)\left(\frac{p}{m_{p}}\right)^{2}\left(\frac{p}{M_{\mathrm{Pl}}}\right)^{n}+\frac{4 \varepsilon p}{m_{p}^{2}}-\frac{[1-(1-\varkappa) A]^{2}}{\varkappa(1-\varkappa)}=0,
$$

where

$$
g(\varkappa)=\frac{1}{A^{2}}-\frac{\varkappa^{n+1}}{(A-1)^{2}}-(1-\varkappa)^{n+1} .
$$

We next consider the threshold configuration for a photon with energy $\varepsilon_{\mathrm{th}}^{\prime} \approx 20 \mathrm{MeV}$. In the rest frame of the Sun, the photon energy is $\varepsilon_{\text {th }}$ and the UHECR is boosted with speed $\beta$ in the direction of Earth. For a head on collision, $\mathbf{k}$ points in the opposite direction, and so the photon energy in the nucleus rest frame is $\varepsilon^{\prime}=\gamma(\varepsilon+\beta k)=\gamma \varepsilon(1+\beta)$. The threshold energy in the rest frame of the Sun is then

$$
\varepsilon_{\mathrm{th}}=\sqrt{\frac{1-\beta}{1+\beta}} \varepsilon_{\mathrm{th}}^{\prime} .
$$

Since $p=\beta E$, we can write

$$
\begin{aligned}
\zeta= & \left(\frac{[1-(1-\varkappa) A]^{2}}{\varkappa(1-\varkappa)}-\frac{4 \beta E \varepsilon_{\mathrm{th}}^{\prime}}{m_{p}^{2}} \sqrt{\frac{1-\beta}{1+\beta}}\right) \\
& \times \frac{\left(\beta E / m_{p}\right)^{-2}\left(\beta E / M_{\mathrm{Pl}}\right)^{-n}}{g(\varkappa)} .
\end{aligned}
$$

We take $E \approx 10^{9.3} \mathrm{GeV}$ and so $\gamma \sim 10^{9}$. With this in mind, we adopt the following expansion:

$$
\sqrt{\frac{1-\beta}{1+\beta}}=\frac{1}{2 \gamma}+\mathcal{O}\left(\frac{1}{\gamma}\right)^{3}
$$

and set $\beta \approx 1$ elsewhere. Substituting (27) into (26) we obtain an expression for the sensitivity of $\zeta$ as a function of $x$,

$\zeta=\left(\frac{[1-(1-\varkappa) A]^{2}}{\varkappa(1-\varkappa)}-\frac{2 A \varepsilon_{\mathrm{th}}^{\prime}}{m_{p}}\right) \frac{\left(m_{p} / E\right)^{2}\left(M_{\mathrm{Pl}} / E\right)^{n}}{g(\varkappa)}$,

where we have used $E=\gamma A m_{p}$. As an illustration, in Fig. 3 we show the sensitivity for probing $\zeta$ as a function of $\varkappa$, assuming observation of a few spatiotemporal coincident showers near the critical energy.

Despite the assumption of Lorentz invariance violation, we want to preserve the timelike character of physical trajectories. For a particle with four momentum $p^{\mu}$, this means that $p_{\mu} p^{\mu}>0$ in a $(+,-,-,-)$ metric signature. Using (17) this condition creates a lower bound $\zeta>\zeta_{\text {null }}$,
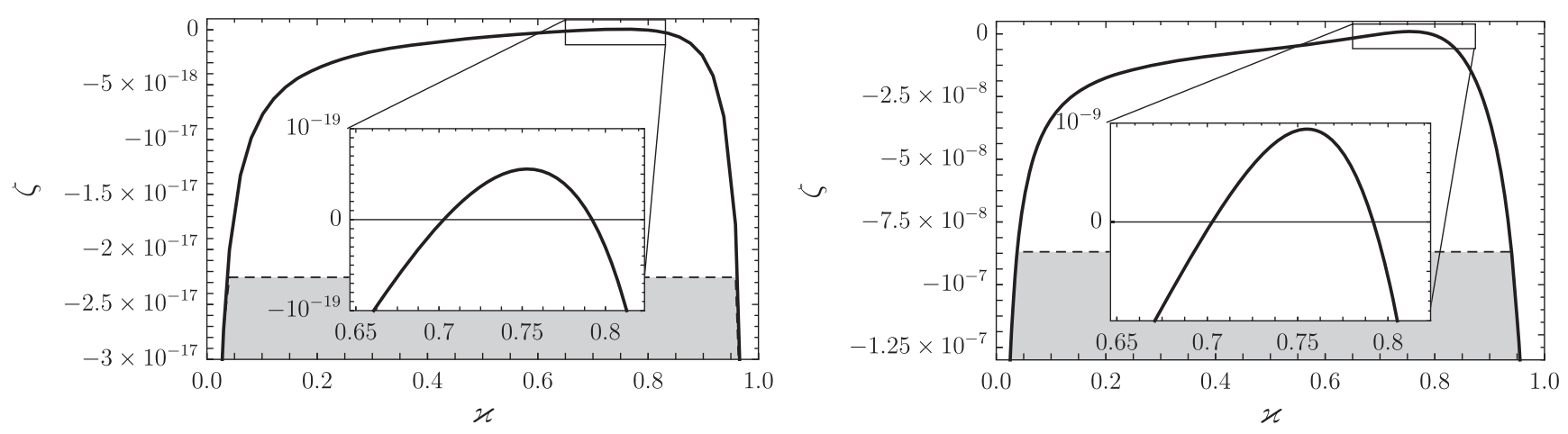

FIG. 3. Sensitivity to $\zeta$ as a function of $\varkappa$ for $n=0$ (left) and $n=1$ (right). We have taken $\varepsilon_{\text {th }}^{\prime}=20 \mathrm{MeV}$ and $E=10^{9.3} \mathrm{GeV}$. The embedded box details the restricted interval of $\varkappa$ for which $\zeta>0$. The shaded band indicates the region for which $\zeta<\zeta_{\text {null }}$. 


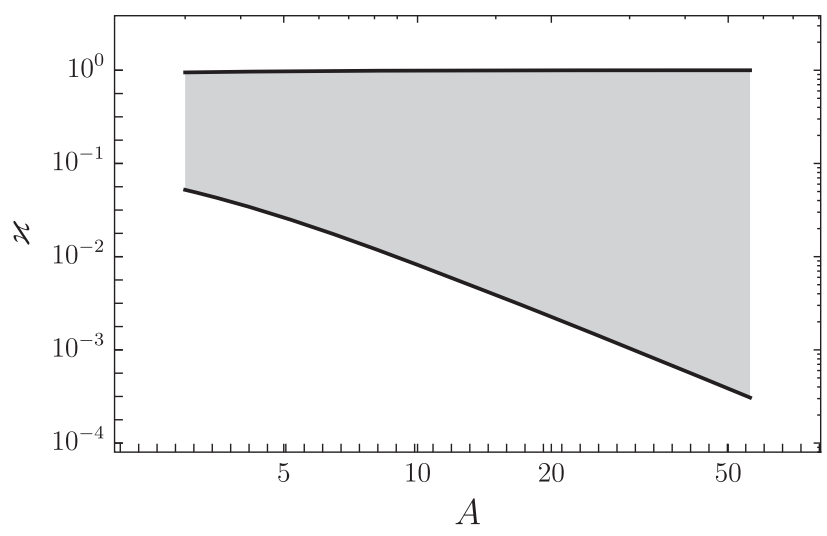

FIG. 4. Allowed (shaded) $\varkappa$ region as a function of $A$, for $n=0$. The lower and upper curves are $\varkappa_{\min }$ and $\varkappa_{\max }$, respectively.

with

$$
\zeta_{\text {null }} \equiv-A^{4}\left(\frac{m_{p}}{E}\right)^{2}\left(\frac{M_{\mathrm{Pl}}}{E}\right)^{n}
$$

assuming $\beta \approx 1$.

The timelike condition is automatically satisfied for positive $\zeta$. In Fig. 3 we show the limiting value $\zeta_{\text {null }}$ and the (shaded) prohibited region. We conclude that with a detection of a few spatiotemporal coincident showers we will be able to constrain $\zeta$ at the level of $\zeta \sim 5 \times 10^{-20}$ for $n=0$, and $\zeta \sim 10^{-9}$ for $n=1$.

Since $g(\varkappa) \leq 0$ for $n=0,1$, using (28) and (29) we can rewrite the timelike condition as

$$
\frac{[1-(1-\varkappa) A]^{2}}{2 A \varkappa(1-\varkappa)}+\frac{1}{2} A^{3} g(\varkappa)<\frac{\varepsilon_{\mathrm{th}}^{\prime}}{m_{p}} .
$$

Using (30) we study the dependence on $A$ and $\varepsilon_{\text {th }}^{\prime}$ of the limiting values $\varkappa_{\min }$ and $\varkappa_{\max }$, such that the timelike condition is satisfied for all $\varkappa_{[}\left[\varkappa_{\min }, \varkappa_{\max }\right]$. Note that near the limits of the interval $\left[\varkappa_{\min }, \varkappa_{\max }\right], d \zeta / d \varkappa$ is large compared to $\varepsilon_{\mathrm{th}}^{\prime} / m_{p}$, for $10 \lesssim \varepsilon_{\mathrm{th}}^{\prime} / \mathrm{MeV} \lesssim 20$ [57]. Thus, the intervals of $\varkappa$ which satisfy (30) barely depend on $\varepsilon_{\text {th }}^{\prime}$, which can be assumed to be zero. For a fixed $n$, the $\varkappa$ limits only depend on $A$. The values of $\varkappa_{\min }$ and $\varkappa_{\max }$ for $n=0$ are shown in Fig. 4. For $n=1$, the values are within a distance of $\sim 10^{-2}$ of those for $n=0$. As can be seen, the values are considerably close to 0 and 1 , with intersections at $[0.04,0.96]$ for $A=4$.

In summary, we have shown that if the photo-disintegration probability of UHECR nuclei on the solar radiation field is $\mathcal{O}\left(10^{-5.5}\right)$, then the unambiguous observation of the extensive air showers that would be produced almost simultaneously by the secondary fragments is within reach of UHECR experiments. This is because our analysis of spatiotemporal correlations indicates that for angular scales $\lesssim 3^{\circ}$ and a time window of $\mathcal{O}(10 \mathrm{~s})$ the signal is background free. Detection of a few events will be enough to constrain Lorentz invariant breaking effects in the range $10^{9} \lesssim \gamma \lesssim 10^{10}$. Such a detection also provides valuable information on the UHECR nuclear composition, which is independent of the hadronic interaction models used to describe the development of air showers, and therefore such information develops complementary to studies of the $X_{\max }$ distribution and its fluctuations.

\section{ACKNOWLEDGMENTS}

We would like to acknowledge many useful discussions with Tom Weiler, Michael Unger, and our colleagues of the Pierre Auger and POEMMA Collaborations. This work has been supported by the U.S. National Science Foundation (NSF) Grant No. PHY-1620661 and by the National Aeronautics and Space Administration (NASA) Grant No. NNX13AH52G.
[1] K. Greisen, End to the Cosmic Ray Spectrum?, Phys. Rev. Lett. 16, 748 (1966).

[2] G. T. Zatsepin and V.A. Kuzmin, Upper limit of the spectrum of cosmic rays, Pis'ma Zh. Eksp. Teor. Fiz. 4, 114 (1966) [J. Exp. Theor. Phys. 4, 78 (1966).

[3] S. R. Coleman and S. L. Glashow, High-energy tests of Lorentz invariance, Phys. Rev. D 59, 116008 (1999).

[4] R. Aloisio, P. Blasi, P. L. Ghia, and A. F. Grillo, Probing the structure of space-time with cosmic rays, Phys. Rev. D 62 , 053010 (2000).

[5] M. Jankiewicz, R. V. Buniy, T. W. Kephart, and T. J. Weiler, Space-time foam and cosmic ray interactions, Astropart. Phys. 21, 651 (2004).
[6] M. Galaverni and G. Sigl, Lorentz Violation in the Photon Sector and Ultrahigh-Energy Cosmic Rays, Phys. Rev. Lett. 100, 021102 (2008).

[7] M. Galaverni and G. Sigl, Lorentz violation and ultrahigh-energy photons, Phys. Rev. D 78, 063003 (2008).

[8] D. M. Mattingly, L. Maccione, M. Galaverni, S. Liberati, and G. Sigl, Possible cosmogenic neutrino constraints on Planck-scale Lorentz violation, J. Cosmol. Astropart. Phys. 02 (2010) 007.

[9] S. T. Scully and F. W. Stecker, Testing Lorentz invariance with neutrinos from ultrahigh-energy cosmic ray interactions, Astropart. Phys. 34, 575 (2011). 
[10] A. Saveliev, L. Maccione, and G. Sigl, Lorentz invariance violation and chemical composition of ultrahigh-energy cosmic rays, J. Cosmol. Astropart. Phys. 03 (2011) 046.

[11] F. W. Stecker, Testing Lorentz symmetry using high energy astrophysics observations, Symmetry 9, 201 (2017).

[12] R. U. Abbasi et al. (HiRes Collaboration), First Observation of the Greisen-Zatsepin-Kuzmin Suppression, Phys. Rev. Lett. 100, 101101 (2008).

[13] J. Abraham et al. (Pierre Auger Collaboration), Observation of the Suppression of the Flux of Cosmic Rays above $4 \times 10^{19} \mathrm{eV}$, Phys. Rev. Lett. 101, 061101 (2008).

[14] T. Abu-Zayyad et al. (Telescope Array Collaboration), The cosmic ray energy spectrum observed with the surface detector of the Telescope Array experiment, Astrophys. J. 768, L1 (2013).

[15] J. Abraham et al. (Pierre Auger Collaboration), Measurement of the energy spectrum of cosmic rays above $10^{18} \mathrm{eV}$ using the Pierre Auger Observatory, Phys. Lett. B 685, 239 (2010).

[16] T. Abu-Zayyad et al. (HiRes-MIA Collaboration), Measurement of the cosmic ray energy spectrum and composition from $10^{17} \mathrm{eV}$ to $10^{18.3} \mathrm{eV}$ using a hybrid fluorescence technique, Astrophys. J. 557, 686 (2001).

[17] J. Abraham et al. (Pierre Auger Collaboration), Measurement of the Depth of Maximum of Extensive Air Showers above $10^{18} \mathrm{eV}$, Phys. Rev. Lett. 104, 091101 (2010).

[18] A. Aab et al. (Pierre Auger Collaboration), Depth of maximum of air-shower profiles at the Pierre Auger Observatory I: Measurements at energies above $10^{17.8} \mathrm{eV}$, Phys. Rev. D 90, 122005 (2014).

[19] A. Aab et al. (Pierre Auger Collaboration), Depth of maximum of air-shower profiles at the Pierre Auger Observatory II: Composition implications, Phys. Rev. D 90, 122006 (2014).

[20] A. Aab et al. (Pierre Auger Collaboration), Evidence for a mixed mass composition at the "ankle" in the cosmic-ray spectrum, Phys. Lett. B 762, 288 (2016).

[21] R. U. Abbasi et al., Study of ultrahigh energy cosmic ray composition using Telescope Array's Middle Drum detector and surface array in hybrid mode, Astropart. Phys. 64, 49 (2015).

[22] R. Abbasi et al. (Pierre Auger and Telescope Array Collaborations), Report of the working group on the composition of ultrahigh energy cosmic rays, J. Phys. Soc. Jpn. Conf. Proc. 9 (2016) 010016.

[23] The significance of this a posteriori study does not account for the previous searches made within the Auger Collaboration and those made by others.

[24] A. Aab et al. (Pierre Auger Collaboration), The Pierre Auger Observatory: Contributions to the 35th International Cosmic Ray Conference (ICRC 2017), arXiv:1708.06592.

[25] A. Aab et al. (Pierre Auger Collaboration), Observation of a large-scale anisotropy in the arrival directions of cosmic rays above $8 \times 10^{18} \mathrm{eV}$, Science 357, 1266 (2017).

[26] R. Aloisio, V. Berezinsky, and P. Blasi, Ultrahigh energy cosmic rays: implications of Auger data for source spectra and chemical composition, J. Cosmol. Astropart. Phys. 10 (2014) 020.

[27] M. Unger, G. R. Farrar, and L. A. Anchordoqui, Origin of the ankle in the ultrahigh energy cosmic ray spectrum, and of the extragalactic protons below it, Phys. Rev. D 92, 123001 (2015).

[28] A. Aab et al. (Pierre Auger Collaboration), Combined fit of spectrum and composition data as measured by the Pierre Auger Observatory, J. Cosmol. Astropart. Phys. 04 (2017) 038.

[29] R. Aloisio, V. Berezinsky, and A. Gazizov, Ultra high energy cosmic rays: The disappointing model, Astropart. Phys. 34, 620 (2011).

[30] S. T. Scully and F. W. Stecker, Lorentz invariance violation and the observed spectrum of ultrahigh energy cosmic rays, Astropart. Phys. 31, 220 (2009).

[31] X. J. Bi, Z. Cao, Y. Li, and Q. Yuan, Testing Lorentz invariance with ultra high energy cosmic ray spectrum, Phys. Rev. D 79, 083015 (2009).

[32] R. Aloisio, D. Boncioli, A. di Matteo, P. L. Ghia, A. F. Grillo, S. Petrera, and F. Salamida, Are cosmic rays still a valuable probe of Lorentz invariance violations in the Auger era?, Frascati Phys. Ser. 58, 274 (2014).

[33] L. A. Anchordoqui, V. Barger, and T. J. Weiler, Cosmic mass spectrometer, J. High Energy Astrophys. 17 (2018) 38.

[34] J. L. Puget, F. W. Stecker, and J. H. Bredekamp, Photonuclear interactions of ultrahigh-energy cosmic rays and their astrophysical consequences, Astrophys. J. 205, 638 (1976).

[35] G. T. Zatsepin, Photofision of heavy cosmic ray particles occuring under the action of solar radiation, Dokl. Akad. Nauk SSSR 80, 577 (1951).

[36] N. M. Gerasimova and G. T. Zatsepin, Disintegration of cosmic ray nuclei by solar photons, Sov. Phys. JETP 11, 899 (1960).

[37] L. A. Anchordoqui, J. F. Beacom, H. Goldberg, S. PalomaresRuiz, and T. J. Weiler, TeV Gamma-Rays from PhotoDisintegration/De-Excitation of Cosmic-Ray Nuclei, Phys. Rev. Lett. 98, 121101 (2007).

[38] S. Karakula and W. Tkaczyk, The formation of the cosmic ray energy spectrum by a photon field, Astropart. Phys. 1, 229 (1993).

[39] G. A. Medina-Tanco and A. A. Watson, The photodisintegration of cosmic ray nuclei by solar photons: The GerasimovaZatsepin effect revisited, Astropart. Phys. 10, 157 (1999).

[40] L. N. Epele, S. Mollerach, and E. Roulet, On the disintegration of cosmic ray nuclei by solar photons, J. High Energy Phys. 03 (1999) 017.

[41] S. Lafebre, H. Falcke, J. Horandel, and J. Kuijpers, Prospects for direct cosmic ray mass measurements through the Gerasimova-Zatsepin effect, Astron. Astrophys. 485, 1 (2008).

[42] J. V. R. van Eijden, S. J. de Jong, and C. J. W. P. Timmermans, Cosmic ray interactions in the solar system: The GerasimovaZatsepin effect, arXiv:1606.07693.

[43] J. R. Hoerandel, On the knee in the energy spectrum of cosmic rays, Astropart. Phys. 19, 193 (2003).

[44] J. Abraham et al. (Pierre Auger Collaboration), Trigger and aperture of the surface detector array of the Pierre Auger Observatory, Nucl. Instrum. Methods Phys. Res., Sect. A 613, 29 (2010).

[45] A. V. Olinto et al., POEMMA: Probe of extreme multimessenger astrophysics, Proc. Sci., ICRC20172017 (2017) 542 . 
[46] A. Aab et al. (Pierre Auger Collaboration), The Pierre Auger Observatory: Contributions to the 33rd International Cosmic Ray Conference (ICRC 2013), arXiv: 1307.5059.

[47] A. N. Gorbunov, V. A. Dubrovina, V. A. Osipova, V. S. Silaeva, and P. A. Cerenkov, Investigation of the photoeffect in light nuclei, J. Exp. Theor. Phys. 15, 520 (1962).

[48] A. N. Gorbunov, Study of the ${ }^{4} \mathrm{He}(\gamma, p){ }^{3} \mathrm{H}$ and ${ }^{4} \mathrm{He}(\gamma, n)^{3} \mathrm{He}$ reactions, Phys. Lett. B 27, 436 (1968).

[49] F. Balestra, E. Bollini, L. Busso, R. Garfagnini, C. Guaraldo, G. Piragino, R. Scrimaglio, and A. Zanini, Photodisintegration of $\mathrm{He}-4$ in the giant resonance region, Nuovo Cimento A 38, 145 (1977).

[50] R. C. McBroom, H. R. Weller, N. R. Roberson, and D. R. Tilley, H-3 $(p, \gamma)$ He-4 reaction below $E=30 \mathrm{MeV}$, Phys. Rev. C 25, 1644 (1982).

[51] J. R. Calarco, S. S. Hanna, C. C. Chang, E. M. Diener, E. Kuhlmann, and G. A. Fisher, Absolute cross section for the reaction $\mathrm{H}-3(p, \gamma)$ He-4 and a review of He-4 $(\gamma, p) \mathrm{H}-3$ measurements, Phys. Rev. C 28, 483 (1983); Erratum, Phys. Rev. C 29, 672E (1984).

[52] R. Bernabei et al., Measurement of the He-4 $(\gamma, p) \mathrm{H}-3$ total cross section and charge symmetry, Phys. Rev. C 38, 1990 (1988).
[53] G. Feldman, M. J. Balbes, L. H. Kramer, J. Z. Williams, H. R. Weller, and D. R. Tilley, H-3 $(p, \gamma)$ He-4 reaction and the $(\gamma, p) /(\gamma, n)$ ratio in He-4, Phys. Rev. C 42, R1167 (1990).

[54] T. Shima, S. Naito, Y. Nagai, T. Baba, K. Tamura, T. Takahashi, T. Kii, H. Ohgaki, and H. Toyokawa, Simultaneous measurement of the photodisintegration of He-4 in the giant dipole resonance region, Phys. Rev. C 72, 044004 (2005).

[55] R. Raut, W. Tornow, M. W. Ahmed, A. S. Crowell, J. H. Kelley, G. Rusev, S. C. Stave, and A. P. Tonchev, Photodisintegration Cross Section of the Reaction He-4 $(\gamma, p) \mathrm{H}-3$ between 22 and $30 \mathrm{MeV}$, Phys. Rev. Lett. 108, 042502 (2012).

[56] W. Tornow, J. H. Kelley, R. Raut, G. Rusev, A. P. Tonchev, M. W. Ahmed, A. S. Crowell, and S. C. Stave, Photodisintegration cross section of the reaction $\mathrm{He}-4(\gamma, n) \mathrm{He}-3$ at the giant dipole resonance peak, Phys. Rev. C 85, 061001 (2012).

[57] F. W. Stecker and M. H. Salamon, Photodisintegration of ultrahigh-energy cosmic rays: A new determination, Astrophys. J. 512, 521 (1999).

[58] D. Mattingly, T. Jacobson, and S. Liberati, Threshold configurations in the presence of Lorentz violating dispersion relations, Phys. Rev. D 67, 124012 (2003). 